\title{
Response of HIV-Associated Proteinuria to Antiretroviral Therapy in HIV-1-Infected Children
}

\section{IraShah}

\author{
Department of Perinatal and Pediatric HIV Clinic, B. J. Wadia Hospital for Children \\ Parel, Mumbai-400012, India
}

\begin{abstract}
HIV-associated nephropathy has been found in children with HIV-1 infection as a late manifestation of this disease; it is associated with nephrotic syndrome with focal segmental glomerulosclerosis and/or mesangial hyperplasia with microcystic tubular dilatation. This is quite rare in children and no cases have been reported from India. Several mechanisms have been hypothesized for the HIVinduced renal damage. We report on two HIV-infected children with HIV-associated proteinuria and dramatic response to antiretroviral therapy.

Key Words: HIV associated nephropathy, HIVAN, HIV associated proteinuria.
\end{abstract}

HIV-associated proteinuria has been found in HIV-infected adult patients; rapid progression to end-stage renal disease is reported [1]. In children infected with HIV-1, renal disease is quite uncommon and onset of proteinuria or azotemia typically occurs two to five years after onset of HIV infection [1]. In a study in the USA, among 7,732 patients with HIV-associated nephropathy, only 60 were younger than 21 years of age [2]. Similarly, in Spain, only three patients out of 395 HIV-infected children had associated renal disease [3]. Till now, there have been no reports of HIV-associated nephropathy in children in India. We report on two children with HIV-associated proteinuria from India and on their response to antiretroviral therapy.

\section{Case 1}

A six-year-old HIV-1-infected boy presented with five days of fever and cough, with one episode of generalized tonic clonic convulsion in the morning. He had been diagnosed to be HIV-infected three years previously and was started on a two-drug antiretroviral therapy (ART), consisting of zidovudine (AZT) and lamivudine (3TC); his parents continued this treatment without regular follow-up by his physician. The boy also had weight loss. Both parents were also HIV-1 infected, suggesting a vertical mode of transmission. His milestones and immunization were up to date. On examination, the patient was tachypneic, with a respiratory rate of $60 / \mathrm{min}$., had hypertension $(\mathrm{BP}=150 / 90 \mathrm{~mm}$ of $\mathrm{Hg})$ and had Grade 3 clubbing with bilateral otorrhoea and oral thrush. He was severely wasted [weight $=12 \mathrm{~kg}\left(<5^{\text {th }}\right.$ percentile $)$ and height $=$ $102 \mathrm{~cm}\left(<5^{\text {th }}\right.$ percentile $\left.)\right]$ and had papular dermatitis. Systemic examination revealed bilateral crepitations. Other systemic examinations were normal. He was suspected to have bronchopneumonia, meningitis, otitis media and hypertension.

Received on 13 June 2006; revised 18 September 2006.

Address for correspondence: Dr.. Ira Shah 240 D.Walkeshwar Road, Malabar Hill,. Mumbai 400006. Phone: 91-22-23693509. E-mail: irashah@pediatriconcall.com.

The Brazilian Journal of Infectious Diseases 2006;10(6):408-410. (C) 2006 by The Brazilian Journal of Infectious Diseases and Contexto Publishing. All rights reserved.
Investigations showed a hemoglobin level of $9.5 \mathrm{gm} / \mathrm{dL}$, a white cell count of $9,200 / \mathrm{mm}^{3}$ (48\% polymorphs, $49 \%$ lymphocytes, $3 \%$ eosinophils), a platelet count of $247,000 /$ $\mathrm{mm}^{3}$ and a raised erythrocyte sedimentation rate (ESR) (110 $\mathrm{mm}$ at the end of 1 hour). CSF showed 10 cells $/ \mathrm{mm}^{3}$ with $4 \%$ polymorphs and 6\% lymphocytes, $48 \mathrm{mg} \%$ sugar and $188 \mathrm{mg} /$ $\mathrm{dL}$ proteins. The CSF cryptococcal latex antigen test was negative. Chest X-rays showed severe bronchopneumonia with hilar lymphadenopathy. The Mantoux test was negative. Arterial blood gas revealed hypoxia without acidosis or alkalosis. Urine examination revealed $3+$ albuminuria on more than three occasions, with a urine albumin/creatinine ratio of 1.55. Other renal function tests were normal. Echocardiography showed left ventricular dilatation, with hypertrophy and a normal ejection fraction (60\%). Ultrasound of the abdomen showed internal echoes in the urinary bladder, with splenic hilum nodes and normal kidney echotexture and size. He was treated with intrevenous antibiotics for 14 days and with nifedipine; the pneumonia did not respond, though blood pressure responded. In view of unresponsive pneumonia, with generalized lymphadenopathy, he was started on antituberculous therapy, because it is the most endemic opportunistic infection in HIV-infected individuals in our country. His fever responded to this treatment. In view of persistent proteinuria, the patient was started on enalapril; albuminuria subsequently subsided. His $\mathrm{CD}_{4}$ count was 450 cells $/ \mathrm{mm}^{3}$, with a $\mathrm{CD}_{4}$ percent of $20 \%$ and a $\mathrm{CD}_{4}: \mathrm{CD}_{8}$ ratio of 0.42 . In view of severe opportunistic infection in the form of pneumonia, HIV-meningitis, cardiomyopathy, proteinuria with hypertension and failure to thrive, his antiretroviral regime was changed to three drugs, consisting of zidovudine, didanosine and efavirenz (a protease inhibitor was not used due to high costs). On follow up, he had a weight gain of $2 \mathrm{~kg}$ in 20 days, with no albuminuria and normal blood pressure.

\section{Case 2}

A two-year three-month-old girl presented with puffy eyes and abdominal distension during the previous month, fever for 15 days, cough for one week and vomiting for three days. She had pneumonia at the age of six months. Her milestones and immunization were appropriate for her age. She had no 
previous hospitalization or blood transfusions. The father had tuberculosis 11 years before and had taken antituberculous drugs for nine months. On examination, she had generalized lymphadenopathy, with bilateral otorrhoea and papular dermatitis. Her weight was $8.1 \mathrm{~kg}\left(5^{\text {th }}-10^{\text {th }}\right.$ percentile $)$ and her height was $84 \mathrm{~cm}\left(10^{\text {th }}-25^{\text {th }}\right.$ percentile). Her blood pressure was normal. Systemic examination revealed hepatosplenomegaly with ascites. Other examination findings were normal. In view of the above clinical features, she was suspected to have acquired immunodeficiency; her HIV-1 ELISA was positive with three different kits. Both parents were also tested for HIV and were found to be infected. Thus she was diagnosed to have vertically-transmitted HIV infection. Her urine albumin was $2+$ on three occasions and her urine albumin/creatinine ratio was 1.4. Her hemogram showed $8.5 \mathrm{gm}$ hemoglobin /dL, a white cell count of $15,700 /$ $\mathrm{mm}^{3}$ (24\% polymorphs, $74 \%$ lymphocytes) with an ESR of 65 $\mathrm{mm}$ at the end of one hour. Her serum albumin was $3.2 \mathrm{gm} / \mathrm{dL}$, with elevated liver transaminases (SGOT $=39 \mathrm{IU} / \mathrm{L}, \mathrm{SGPT}=80$ IU/L) and normal cholesterol, BUN, serum creatinine and electrolytes. Chest X-rays revealed miliary tuberculosis (Mantoux test was negative), and ultrasound of the abdomen showed hepatosplenomegaly with coarse echotexture of the liver. HBsAg and Anti HCV were negative. Fine needle aspiration of lymph nodes showed reactive hyperplasia. Thus, she was diagnosed to have HIV infection with tuberculosis, proteinuria, anemia and liver dysfunction. Her $\mathrm{CD}_{4}$ count was 977 cells $/ \mathrm{mm}^{3}$ with a $\mathrm{CD}_{4}$ percent of $15.09 \%$ and a $\mathrm{CD}_{4}: \mathrm{CD}_{8}$ ratio of 0.2. In view of her clinical presentation and immune suppression she was started on antituberculous therapy and a three-drug antiretroviral therapy (ART) consisting of zidovudine, lamivudine and nevirapine. She was given oral amoxicillin for seven days for otitis media. Fifteen days after starting this treatment, her ascitis had resolved, liver enzymes were normal, her weight had increased by $1 \mathrm{~kg}$ and proteinuria had disappeared. On follow-up after two months, she had a weight gain of $2 \mathrm{~kg}$ and she had no proteinuria and a chest Xray showed clearing of miliary shadows.

\section{Discussion}

HIV-associated nephropathy is typically a late manifestation of AIDS [1]. Several mechanisms for pathogenesis have been implicated. HIV-1 virus may directly affect the growth and differentiation of glomerular and tubular epithelial cells, increase recruitment of infiltrating mononuclear cells and cytokines, and up-regulate renal heparan sulphate proteoglycans [1]. Other agents that can lead to associated renal disease are opportunistic infections, nephrotoxic agents and immunological abnormalities [4]. HIV-associated nephropathy presents with heavy proteinuria and usually is seen with other AIDS-related illnesses, such as encephalopathy and cardiomyopathy [1]. Both of our patients presented with massive proteinuria, though not in the nephrotic range, and they had other organ dysfunctions in the form of cardiomyopathy and hypertension in one patient and failure to thrive in both. In addition, both also had tuberculosis. Thus both patients had severe HIV disease. Hypertension may be seen in patients with long-standing HIVassociated nephropathy [1]. A study from Washington found that early stages of HIV-associated nephropathy (HIVAN) in children are associated with enlarged echogenic kidneys, proteinuria and urine microcysts. Also progression to endstage renal disease is slow in children [5]. Both her patients only had proteinuria, and ultrasound of the kidneys was normal; probably HIVAN was at a very early stage. Several studies have found that HIVAN is seen with low $\mathrm{CD}_{4}+$ counts and increased viral loads [6,7]. Similarly both our patients were immunologically affected and were in immune category 2. HIVAN has been treated with various treatment regimes, including HAART [8], angiotensin-converting enzyme inhibitors (ACE inhibitors) [9], and steroids [10], among which HAART has been the most promising. In fact, it was only in 1999 that the first response to HIV-associated nephrotic syndrome in a child with triple dry ART was reported [8]. Similarly, both her patients were started on threedrug ART, with resolution of proteinuria within a few weeks. Though both her patients were immunosuppressed at the onset and attained resolution only after starting ART, it appears that there was either an immune reconstitution or a decrease in the HIV-viral load itself. Immune reconstitution syndrome following HAART in HIV-infected patients is characterized by inflammatory worsening of organ functions, despite improvement in HIV surrogate markers of HIV infection. Jehle et al. described an HIV-infected patient with miliary tuberculosis and urinary shedding of acid-fast bacilli who developed acute renal failure eight weeks after initiation of antituberculosis therapy and six weeks after initiation of HAART; they concluded that immune reconstitution syndrome was the cause of his renal disease [11]. Thus, the response in our patients may have been due to a decrease in HIV-viral load due to ART rather than to immune reconstitution.

We conclude that HIV nephropathy is rare in children. Proteinuria is one of the earliest signs of HIVAN, and early triple-antiretroviral therapy prevents progression of HIVAN.

\section{Acknowledgements}

The authors thank Dr. Dod, Chief Senior Executive of B. J. Wadia Hospital for Children for giving permission to publish this article.

\section{References}

1. Ray P.E., Xu L., Rakusan T., Liu X.H. A 20 years history of childhood HIV-associated nephropathy. Pediatr Nephrol 2004; 19:1075-92. 
2. Ahuja T.S., Abbott K.C., Pack L., Kuo Y.F. HIV-associated nephropathy and end-stage renal disease in children in the United States. Pediatr Nephrol 2004;19:808-11.

3. No authors listed. HIV-related nephropathy in children: The situation in Spain. An Esp Pediatr 1993;39:489-92.

4. Rao T.K.S., Friedman E.A., Nicastri A.D. The types of renal disease in the acquired immunodeficiency syndrome. N Engl J Med 1987;316:1062-8.

5. Ray P.E., Rakusan T., Loechelt B.J., et al. Human immunodeficiency virus (HIV) - associated nephropathy in children from the Washington, D.C. area: 12 years' experience. Semin Nephrol 1998; 18:396-405.

6. Krawczyk C.S., Holmberg S.D., Moorman A.C., et al. HIV outpatient Study Group. Factors associated with chronic renal failure in HIV-infected ambulatory patients. AIDS 2004; $18: 2171-8$.
7. Szezech L.A., Hoover D.R., Feldman J.G., et al. Association between renal disease and outcomes among HIV-infected women receiving or not receiving antiretroviral therapy. Clin Infect Dis 2004;39:1199-206.

8. Viani R.M., Danker W.M., Muelenaer P.A., Spector S.A. Resolution of HIV-associated nephropathy nephrotic syndrome with highly active antiretroviral therapy delivered by gastrostomy tube. Pediatrics 1999;104:1394-6.

9. Kimmer P.L., Mishkin G.J., Umana W.O. Captopril and renal survival in patients with human immunodeficiency virus nephropathy. AM J Kidney Dis 1996;28:202-8.

10. Eustace J.A., Nuermberger E., Choi M., et al. Cohort study of the treatment of severe HIV-associated nephropathy with corticosteroids. Kidney Int 2000;58:1253-60.

11. Jehle A.W., Khanna N., Sigle J.P., et al. Acute renal failure on immune reconstitution in an HIV-positive patient with miliary tuberculosis. Clin Infect Dis. 2004;38(4):e32-5. 